\title{
Propriedades Mecânicas e Morfologia de Blendas de Polipropileno com TPEs
}

\author{
Flávia 0. M. S. Abreu, Maria Madalena de C. Forte \\ Escola de Engenharia, UFRGS
}

Susana A. Liberman Braskem S.A

\begin{abstract}
Resumo: Blendas de polipropileno e elastômeros termoplásticos (TPEs), estireno-b-butadieno-b-estireno (SBS) e estirenob-etileno-co-butileno-b-estireno(SEBS) foram preparadas com o objetivo de avaliar a influência do tipo e da concentração do elastômero nas propriedades mecânicas e na morfologia das blendas. Foram utilizados dois tipos de polipropileno, um homopolímero de propileno (PP-H) e um copolímero randômico de propileno-etileno (PP-R), sendo avaliado também o efeito das características da matriz termoplástica. O elastômero termoplástico aumentou a resistência ao impacto do PP, e a variação da rigidez das blendas foi dependente somente da quantidade de TPE adicionada, sendo estas comparativamente mais rígidas que aquelas com igual teor de elastômero convencional, tipo EPDM e EPR. A blenda com melhor balanço rigidez-impacto foi aquela de PP-R com $10 \%$ de SEBS. As blendas do copolímero de propileno-etileno com os TPEs apresentaram maior deformação do que aquelas com o homopolímero, devido à natureza menos cristalina da matriz do copolímero de propileno. As blendas tanto do homo quanto do copolímero de propileno com SEBS ficaram mais homogêneas em função da maior afinidade do bloco central poliolefínico EB (etileno-co-butileno) do primeiro com a região amorfa da matriz, sendo esta mais significativa no PP-R.
\end{abstract}

Palavras-chave: Polipropileno, copolímeros em bloco, propriedades mecânicas, morfologia.

\section{Morphology and Mechanical Properties of Polypropylenes/TPEs Blends}

Abstract: Blends of polypropylene and thermoplastic elastomers (TPEs) of styrene-butadiene-styrene (SBS) and styreneethylene-co-butene-styrene (SEBS) triblock copolymers were prepared to evaluate the effect of the elastomer and its concentration on the material properties. For this purpose, a polypropylene homopolymer ( $\mathrm{PP}-\mathrm{H})$ and a propylene-ethylene random copolymer (PP-R) were used to evaluate the matrix effect on the tensile properties and morphology of the blends. The addition of TPEs to PP promotes increase on impact resistance and the PP-R/SEBS 10\%wt blend showed the best balance in stiffness-impact resistance. The morphology of binary blends was similar to elastomeric domains dispersed into the PP matrix and changed as the TPE type and concentration varied. The size of SEBS domains was more homogeneous than the SBS' in both PP matrices due to the high affinity between the polyolefinic EB (ethylene-butene) middle block of the SEBS with the PP amorphous fraction.

Keywords: Polypropylene, block copolymers, blends, mechanical properties, morphology.

\section{Introdução}

O polipropileno pela sua versatilidade tem sido um dos materiais commodities mais amplamente utilizado. Entretanto o polipropileno (PP) é um material pouco tenaz com relativamente baixa resistência ao impacto, sendo necessário em algumas de suas aplicações a adição de modificadores de impacto. A adição de elastômeros ao PP, gerando uma fase elastomérica neste, tem sido uma solução economicamente viável, com resultados altamente satisfatórios ${ }^{[1]}$. Nas blendas de polipropileno com elastômeros, aqueles de natureza olefínica, como as borrachas etileno-co-propileno-co-dieno (EPDM $)^{[2,3]}$ e etileno- propileno (EPR) ${ }^{[4-6]}$, têm sido os modificadores de impacto mais usuais. Com o desenvolvimento de novas tecnologias e produção de novos polímeros elastoméricos, os copolímeros de etileno e alfa-olefinas ${ }^{[7-9]}$ e os elastômeros termoplásticos (TPEs) passaram a ser usados como tenacificadores ${ }^{[10-15]}$ ou agentes compatibilizantes $^{[16,17]}$ em blendas com polipropileno. Os TPEs compreendem uma classe de polímeros que, devido a sua estrutura ou morfologia, apresentam propriedades físicas semelhantes à dos elastômeros, sendo processáveis pelos mesmos métodos de transformação empregados para os termoplásticos ${ }^{[18]}$. Os copolímeros tribloco com estrutura do tipo A-B-A_são um tipo de elastômero termoplástico,

Autor para correspondência: Maria Madalena de C. Forte, Escola de Engenharia, Departamento de Materiais, Laboratório de Materiais Poliméricos, UFRGS, Av. Bento Gonçalves 9500, Bairro Agronomia, CEP: 91501-970, Porto Alegre, RS. E-mail: mmcforte@ufrgs.br 
constituídos por um bloco central flexível, e por blocos laterais termoplásticos rígidos ou passíveis de cristalização. Blendas de PP com TPEs tipo copolímero tribloco podem apresentar propriedades diferenciadas daquelas com elastômeros convencionais, como melhor tenacidade com pouca diminuição dos valores de módulo elástico ${ }^{[14,19]}$. A adição de elastômeros convencionais ao PP, por sua vez, reduz significativamente a sua rigidez limitando o teor de elastômero na composição da blenda. Para compensar este efeito e melhorar as propriedades mecânicas, cargas como talco e carbonato de cálcio ${ }^{[10]}$, mica e fibra de vidro tem sido adicionadas ao PP com produção de materiais com valores de propriedades da ordem daquelas de plásticos de engenharia ${ }^{[20]}$.

Blendas de polipropileno (PP) com estireno-b-butadienob-estireno (SBS) e estireno-b-etileno-co-butileno-b-estireno (SEBS) foram preparadas com o objetivo de avaliar o efeito desses elastômeros nas propriedades mecânicas da blenda. Para verificar a influência da natureza química do polipropileno, utilizou-se um homopolímero de propileno de alta cristalinidade, o polipropileno isotático (PP-H), e um copolímero de propileno-etileno (PP-R) com menor cristalinidade e, portanto com maior resistência ao impacto. Em trabalho anterior ${ }^{[21]}$, foi discutido principalmente o efeito dos TPEs estirênicos como agente de nucleação para o polipropileno, através da avaliação do comportamento térmico das blendas por DSC. Observou-se um deslocamento do pico de cristalização do polipropileno para temperaturas superiores na presença de ambos TPEs, sendo que o SBS foi mais efetivo que o SEBS como nucleante. A influência e a efetividade relativa desses, como agente de nucleação, foi discutida em função da natureza química do bloco central (polibutadieno ou poli(etileno-co-butileno)) e rigidez dos blocos laterais de poliestireno. As blendas de PP com SEBS apresentaram domínios elastoméricos menores e maior resistência ao impacto. Neste trabalho, o comportamento mecânico das blendas de PP com SEBS ou SBS com teores de TPE de 10 a 30\%, será correlacionado com a natureza química e morfologia destas.

\section{Experimental}

Materiais: Foram utilizadas amostras comerciais de polipropileno isotático $(\mathrm{PP}-\mathrm{H})($ solúveis em xileno $=4,0 \%) \mathrm{e}$ de copolímero randômico de propileno-etileno (PP-R) (solúveis em xileno $=6,5 \%$ ) com $3 \%$ em peso de eteno, ambos produzidos pela Braskem S.A. Os elastômeros termoplásticos utilizados, doados gentilmente pela Kraton S. A, foram os copolímeros em bloco estireno-b-butadieno-b-estireno (SBS) e estireno-b-etileno-co-butileno-b-estireno (SEBS) ambos com $30 \%$ de estireno. A Tabela 1 mostra os valores de índice de fluidez, de viscosidade complexa, de massa molar e distribuição de massas molares dos polímeros, bem como a cristalinidade dos polipropilenos ${ }^{[21]}$.

Preparação das blendas: A mistura dos grânulos dos componentes das blendas foi homogeneizada em um misturador de sólidos por $3 \mathrm{~min}$ sob a rotação de $1.500 \mathrm{rpm}$. Em segui-
Tabela 1. Características dos PPs e TPEs utilizados

\begin{tabular}{lcccc}
\hline & \multicolumn{2}{c}{ Polipropileno } & \multicolumn{2}{c}{ TPE } \\
\cline { 2 - 5 } & PP-H & PP-R & SBS & SEBS \\
\hline MI (g/10min) & $11,6^{\mathrm{a}}$ & $10,7^{\mathrm{a}}$ & $<1,0^{\mathrm{b}}$ & $<1,0^{\mathrm{b}}$ \\
$\eta$ (Pa. s 10 Hz) & 632 & 660 & 25.388 & 35.308 \\
$\overline{\mathrm{M}} \mathrm{w}(\mathrm{g} / \mathrm{mol})$ & 236.150 & 251.850 & 92.610 & 72.360 \\
$\overline{\mathrm{M}} \mathrm{w} / \mathrm{M} \mathrm{n}$ & 4,4 & 4 & 1,4 & 1,1 \\
Cristalinidade (\%) & $56^{\mathrm{c}}$ & $38^{\mathrm{c}}$ & amorfo & amorfo
\end{tabular}

a: $230^{\circ} \mathrm{C}$ e $2,16 \mathrm{~kg}$; b: $200{ }^{\circ} \mathrm{C}$ e $5 \mathrm{~kg}$ c:estimada a partir do calor de fusão $(\Delta \mathrm{Hm})$ por DSC (incerteza da medida $3 \%$ ).

$\mathrm{da}_{2}$ a mistura dos polímeros no estado fundido foi feita em uma extrusora rosca simples, marca Oryzon, a $60 \mathrm{rpm}$, com perfil de temperatura igual a $200{ }^{\circ} \mathrm{C} / 220{ }^{\circ} \mathrm{C} / 220^{\circ} \mathrm{C} / 230{ }^{\circ} \mathrm{C} /$ $230^{\circ} \mathrm{C}$. As composições das blendas variaram de 5 a $30 \%$ de TPE. Os corpos de prova utilizados nos ensaios mecânicos e na avaliação da morfologia das blendas foram preparados em uma máquina injetora Battenfeld 240.

Cromatografia de exclusão por tamanho: Os polipropilenos (PP-H e PP-R) e copolímeros estirênicos (SBS e SEBS) foram analisados por cromatografia de exclusão por tamanho (Waters, modelo 150C) em 1,3,5-triclorobenzeno a $140{ }^{\circ} \mathrm{C}$. Foram utilizados detector de índice de refração e viscométrico e colunas Toso-Haas do tipo mixed cobrindo a faixa de massa molar de 1.000 a $8.500 .000 \mathrm{~g} / \mathrm{mol}$. As massas molares médias ponderal $(\bar{M} w)$ e numérica média $(\bar{M} n)$ foram determinadas utilizando-se curva de calibração universal de padrões de poliestireno, polipropileno e polietileno. As análises foram realizadas em duplicata, onde foi considerado o valor médio dos resultados. O desvio padrão dos resultados de $(\bar{M} w)$ e $(\bar{M} n)$ das amostras foi menor que $8 \%$.

Avaliação morfológica: A morfologia das blendas foi avaliada através de um microscópio eletrônico de varredura Jeol JSM 5800 com voltagem de aceleração de $20 \mathrm{kV}$. Os corpos de prova fraturados, após resfriamento por 2 minutos em nitrogênio líquido, foram imersos em tetrahidrofurano a $60^{\circ} \mathrm{C}$ por 30 min para extração das partículas elastoméricas da superfície fraturada. Após seca, a superfície fraturada foi recoberta por uma fina camada de ouro em um metalizador. O tamanho médio dos domínios do TPE na blenda foi determinado usando-se um software analisador de imagens Leica.

Propriedades de tração: As propriedades mecânicas dos polipropilenos e de suas blendas com TPEs foram avaliadas em uma máquina universal de ensaios Instron modelo 4202, de acordo com a norma ASTM D 638-95, utilizando-se velocidade de teste de $2 \mathrm{~mm} / \mathrm{min}$. A incerteza da medida no teste de tração para tensão no escoamento e na ruptura é $6 \%$, enquanto que para módulo é cerca de 3\%. Para os resultados de elongação no escoamento e na ruptura a incerteza é de cerca de 10 e $30 \%$, respectivamente.

Propriedade de impacto: A resistência ao impacto Izod, à temperatura ambiente $\left(23^{\circ} \mathrm{C}\right)$ e a baixa temperatura $\left(0{ }^{\circ} \mathrm{C}\right)$, de corpos de prova das amostras com entalhe foi determinada 
em uma máquina de impacto CEAST modelo 6845-800 de acordo com norma ASTM D 256. A incerteza da medida no teste de impacto é de cerca de $10 \%$.

\section{Resultados e Discussão}

Os polipropilenos utilizados neste trabalho apresentam diferenças quanto a sua natureza química, considerando que o PP-H é um homopolímero de propileno e o PP-R é um copolímero de propileno e etileno. O PP-H possui alto índice de isotaticidade (medida da estereorregularidade da cadeia), uma vez que apresenta teor de solúveis em xileno igual a 4,0\%. Esta fração solúvel em xileno é constituída principalmente por cadeias de PP com configuração atática, não estereorregular ou fração de PP de baixa massa molar. A introdução de etileno na cadeia do polipropileno baixa sua cristalinidade e conseqüentemente, eleva a fração de solúveis em xileno que no caso do PP-R é igual a 6,5\%. Esta diferença na composição de ambos manifesta-se nas propriedades mecânicas, sendo que o PP-H apresenta maior módulo e rigidez e menor resistência ao impacto que o PP-R. Os elastômeros termoplásticos SBS e SEBS são copolímeros tribloco lineares, sendo os blocos laterais poliestireno (PS) e, portanto, rígidos à temperatura ambiente, e o bloco central polibutadieno (B) e poli(etileno-co-butileno) (EB), respectivamente, flexíveis. O bloco EB é uma poliolefina, portanto com característica química e estrutural semelhante à dos polipropilenos, como pode ser visualizado na Figura 1. A natureza olefínica do bloco central do SEBS e do polipropileno pode favorecer a interação e dispersão do elastômero na matriz do PP, gerando morfologia e propriedades diversas daquelas obtidas com o SBS.

As amostras de PP foram selecionadas com índice de fluidez, massa molar ponderal média $(\bar{M} w)$, polidispersão

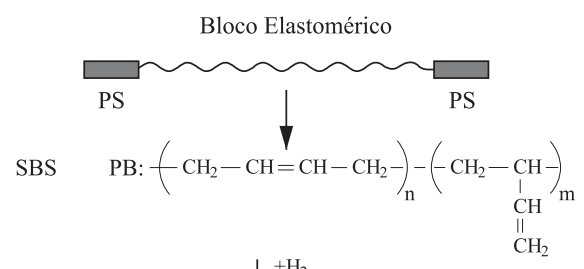

(a)

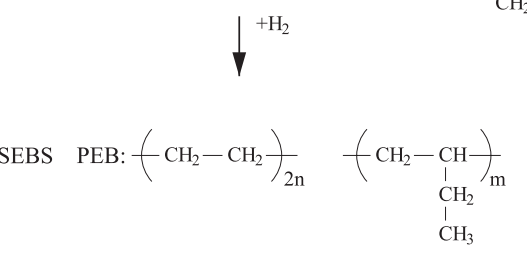

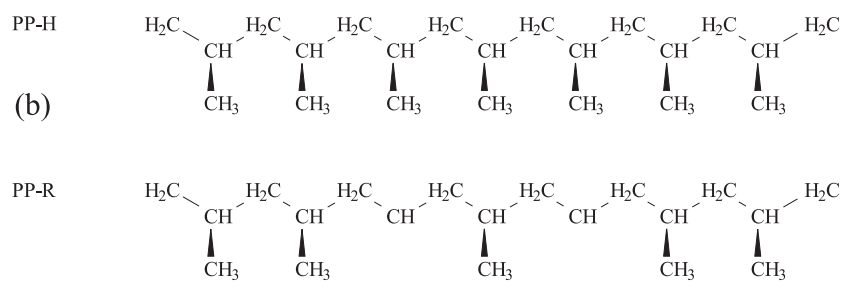

Figura 1. Esquema simplificado da estrutura química dos (a) TPEs (SBS e SEBS) e dos (b) PPs (PP-H e PP-R).
$(\bar{M} w / \bar{M} n)$ e viscosidade complexa $\left(\eta^{*}\right)$ da mesma ordem de grandeza (vide Tabela 1). Assim, a diferença entre os polipropilenos consistiu basicamente na sua composição química e cristalinidade, sendo que esta última, determinada por DSC, foi de aproximadamente 56 e 38\% para o homopolímero (PP-H) e copolímero (PP-R), respectivamente, tendo o PP-R maior fração amorfa. O índice de fluidez dos TPEs foi inferior a 1,0g/10 min, e a massa molar ponderal média $(\bar{M} w)$ destes foi da mesma ordem de grandeza, sendo polímeros monodispersos. A razão entre a viscosidade complexa do TPE e a do PP $\left(\eta{ }^{*}{ }_{\mathrm{TPE}} / \eta^{*}{ }_{\mathrm{PP}}\right)$ foi da ordem de 40 e 55 , respectivamente, para as blendas de PP com SBS e SEBS (Tabela 1). A ordem de grandeza da razão $\eta^{*}{ }_{\mathrm{TPE}} / \eta^{*}{ }_{\mathrm{PP}}$ foi significativamente alta, e a diferença deu-se entre estas em função do elastômero. Uma vez que as massas molares tanto dos polipropilenos (PP-H e do PP-R) como dos elastômeros termoplásticos (SBS e SEBS) não eram significativamente diferentes entre si, possíveis efeitos destas na mistura foram desconsiderados frente à diferenciação existente na estrutura química dos polímeros. A eficiência do SBS e SEBS em modificar as propriedades mecânicas dos polipropilenos com diferentes graus de cristalinidade foi correlacionada a eventuais diferenças morfológicas do material observada por MEV.

\section{Morfologia das blendas PP/TPE}

A morfologia da mistura de polímeros depende das propriedades interfaciais e reológicas dos componentes, sendo que a adesão interfacial entre o elastômero e a matriz influencia significativamente as propriedades mecânicas da blenda $^{[7,11,12]}$. Em blendas imiscíveis, a fase dispersa apresenta domínios com tamanho e forma diferenciados em virtude da quantidade relativa entre seus componentes e da razão entre as viscosidades da matriz e do elastômero. A razão entre as viscosidades da matriz e do elastômero é determinante da morfologia final gerada no processamento do fundido. Quando esta razão é muito alta, pode haver um aumento no tamanho dos domínios da fase dispersa devido a coalescência de partículas do elastômero. A diminuição da viscosidade da matriz reduz a habilidade desta em transferir tensões de cisalhamento para a fase elastomérica, causando sua ruptu$\mathrm{ra}^{[22]}$. Como observado em outras blendas de PP modificadas com elastômeros ${ }^{[1,23-25]}$, aquelas de PP/TPE preparadas neste trabalho apresentaram morfologias distintas com domínios de elastômero dispersos na matriz de PP. A Figura 2 apresenta as micrografias de $\mathrm{MEV}$ das blendas do homopolímero (PP-H) com 10 e 30\% de SBS e com 10 e $30 \%$ de SEBS, nas quais os domínios escuros correspondem ao espaço anteriormente ocupado pelo TPE. A morfologia das blendas dependeu do teor de TPE e principalmente naquelas com maiores teores, a forma, tamanho e distribuição dos domínios variaram conforme o tipo da matriz e TPE. Nas blendas de PP-H com 10\% de TPE, não houve diferença significativa entre os domínios elastoméricos para os dois tipos de TPE, como pode ser visto na Figura 2 (a) e (c). No entanto, quando o teor de TPE foi de 30\%, os domínios de SEBS (Figura 2 (d)) foram menores e melhor dispersos com- 

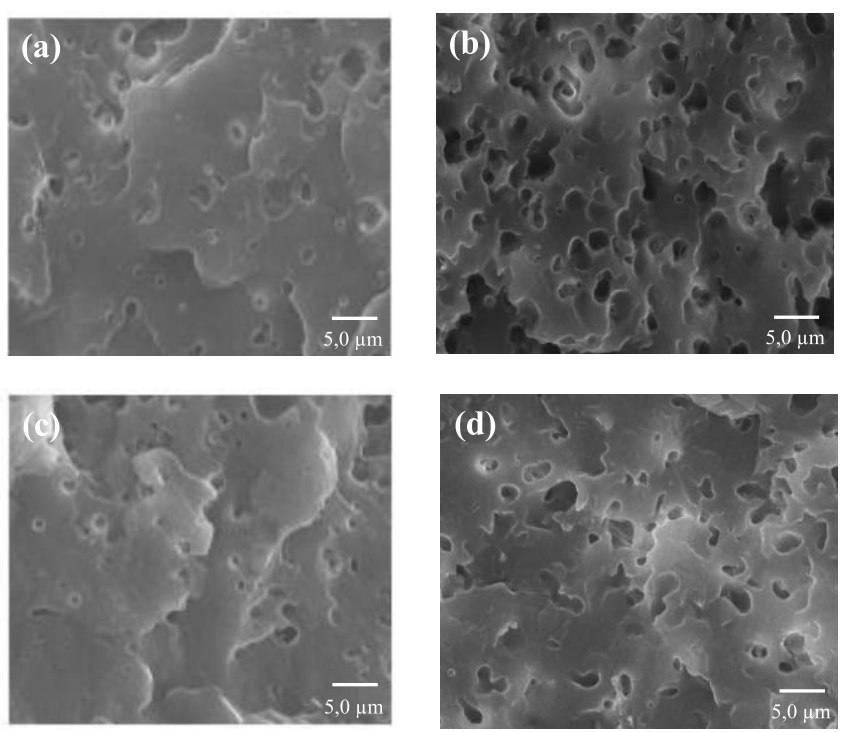

Figura 2. Micrografias das blendas de PP-H/TPE com (a) 10 e (b) $30 \%$ de SBS e (c) 10 e (d) $30 \%$ de SEBS (3000x).

parados aqueles de SBS (Figura 2 (b)). Pode-se afirmar, por inferência, que os domínios elastoméricos maiores se formam pela coalescência dos menores. A melhor dispersão do SEBS na matriz do homopolímero (PP-H), comparativamente ao SBS, pode ser atribuída à existência de maior afinidade do bloco central poliolefínico (EB) com o polipropileno em relação aquele butadiênico.

A Figura 3 mostra micrografias de MEV das blendas do copolímero PP-R com 10 e 30\% de SBS e com 10 e $30 \%$ de SEBS. Para blendas com $10 \%$ de TPE, assim como observado para as de PP-H, não houve variação na morfologia da blenda em função do tipo do elastômero (Figura 3 (a) e (c)). Quando o teor de TPE na blenda foi de 30\%, os domínios tornam-se menores e melhor definidos (Figura 3 (b) e (d)). Comparando-se a morfologia das blendas, com $10 \%$ de TPE, é possível observar que as de PP-R (Figuras 3 (a) e (c)) apresentaram menor quantidade de domínios do que as de PP-H (Figuras 2 (a) e (c)). A blenda de PP-R/SBS30\% (Figura 3 (b)) apresentou domínios com formato esférico e distribuição mais homogênea, indicando um menor grau de coalêscencia do que na blenda PP-H/SBS30\% (Figura 2 (b)). O diâmetro médio das partículas dos elastômeros foi da ordem de $2,34 \mu \mathrm{m}$ para a blenda PP-H/SBS e 1,25 $\mu \mathrm{m}$ para a blenda PP-R/SBS, conforme discutido em trabalho anterior ${ }^{[21]}$.

\section{Resistência ao Impacto das blendas PP/TPE}

A resistência ao impacto de um polímero semicristalino pode ser modificada através da adição de elastômero neste. No entanto, essa varia significativamente com a composição e a morfologia final da blenda. As diferenças morfológicas das blendas de PP/TPEs, conforme verificado pelo tamanho médio dos domínios dos elastômeros na matriz, foram relacionadas com os resultados de resistência ao impacto. Ambos os polipropilenos, $\mathrm{PP}-\mathrm{H}$ e PP-R, no teste de impacto Izod, nas temperaturas de zero e $23{ }^{\circ} \mathrm{C}$, apresentaram fratura frágil e diferente capacidade de absorção de energia, sendo a resis-
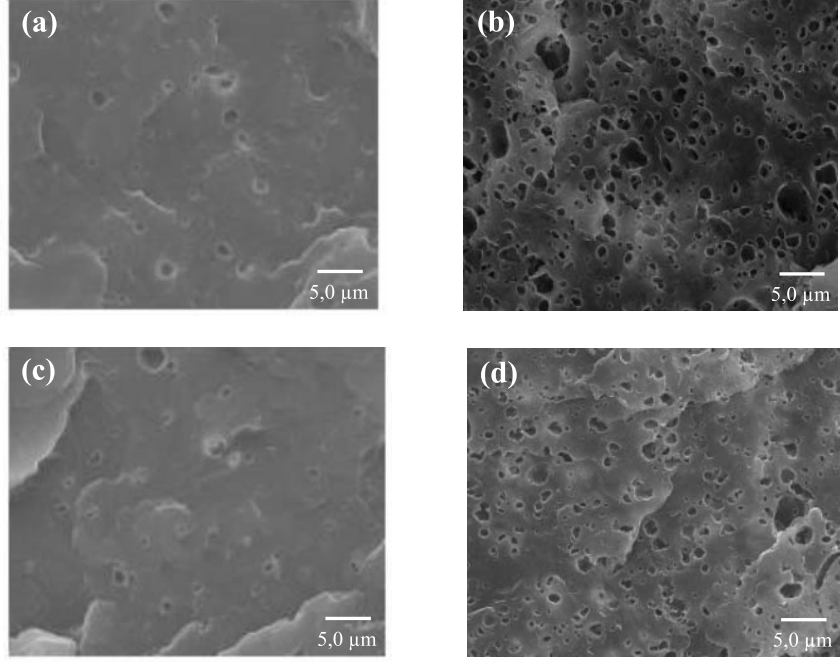

Figura 3. Micrografias das blendas de PP-R/TPE com (a) 10 e (b) $30 \%$ de SBS e (c) 10 e (d) 30\% de SEBS (3000x).

tência ao impacto a $23{ }^{\circ} \mathrm{C}$ iguais a 35 e $51 \mathrm{~J} / \mathrm{m}$ para o $\mathrm{PP}-\mathrm{H}$ e PP-R, respectivamente. A adição de TPE em ambos tipos de PP aumentou a resistência ao impacto destes em função tanto do teor como do tipo de elastômero. A Tabela 2 mostra os valores de resistência ao impacto Izod das blendas de polipropileno a 23 e a $0{ }^{\circ} \mathrm{C}$, bem como o tamanho médio dos domínios dos elastômeros na matriz dos polipropilenos (PPH e PP-R). A adição de 10\% de TPE em ambos PP aumentou em mais de $30 \%$ a resistência ao impacto destes. O tamanho médio dos domínios de TPE variou entre 1,2 e 2,4 $\mu \mathrm{m}$, sendo os domínios de SBS sempre maiores do que os de SEBS, principalmente nos materiais com maiores teores de TPE.

A blenda PP-H/SBS30\% apresentou resistência ao impacto a $23{ }^{\circ} \mathrm{C}$ cerca de sete vezes maior que aquela do PP homopolímero, enquanto para a com igual teor de SEBS não houve fratura dos corpos de prova. Essa diferença na resposta mecânica das blendas PP-H/TPE 30\% com SBS ou SEBS pode ser associada à morfologia dessas, já que os domínios de SEBS ficaram mais bem dispersos e menores $(1,6 \mu \mathrm{m})$ do que os de $\operatorname{SBS}(2,3 \mu \mathrm{m})$.

As blendas PP-R/TPE apresentaram comportamento similar ao das blendas de PP-H/TPE, ou seja, a resistência ao impacto em ambas temperaturas aumenta com o teor de TPE na mistura, e em blendas com até $20 \%$ de TPE o valor da resistência ao impacto foi o dobro do obtido em relação ao PP-R puro. Um teor de $30 \%$ de SBS na blenda com PP-R eleva cerca de nove vezes o valor da resistência ao impacto a $23{ }^{\circ} \mathrm{C}$, enquanto para aquela com igual teor de SEBS não houve fratura do corpo de prova. A diferença na resposta mecânica das blendas de PP-R/TPE30\% não pode ser interpretada somente como conseqüência da morfologia dos domínios elastoméricos da matriz, os quais foram similares e da ordem de 1,2 e 1,3 $\mu \mathrm{m}$, respectivamente, para as blendas com SEBS e SBS, mas também devido à natureza química dos elastômeros.

O tamanho dos domínios da fase elastomérica dispersos em uma mesma matriz pode ser relacionado com a razão das 
Tabela 2. Resistência ao impacto Izod $\left(23\right.$ e $\left.0^{\circ} \mathrm{C}\right)$ e tamanho de partícula da fase dispersa das blendas de PP/TPE

\begin{tabular}{|c|c|c|c|c|c|c|}
\hline \multirow{3}{*}{$\begin{array}{c}\text { Blenda } \\
\text { (\% de elastômero) }\end{array}$} & \multicolumn{4}{|c|}{ SBS } & \multirow{2}{*}{\multicolumn{2}{|c|}{$\begin{array}{c}\text { SEBS } \\
\text { Impacto Izod } \\
(\mathrm{J} / \mathbf{m})\end{array}$}} \\
\hline & \multirow[t]{2}{*}{$\begin{array}{l}\text { Tamanho de } \\
\text { partícula } \\
(\mu \mathrm{m})\end{array}$} & \multicolumn{2}{|c|}{$\begin{array}{c}\text { Impacto Izod } \\
(\mathbf{J} / \mathbf{m})\end{array}$} & \multirow[t]{2}{*}{$\begin{array}{l}\text { Tamanho de } \\
\text { partícula } \\
(\mu \mathrm{m})\end{array}$} & & \\
\hline & & $23^{\circ} \mathrm{C}$ & $\mathbf{0}^{\circ} \mathrm{C}$ & & $23^{\circ} \mathrm{C}$ & $\mathbf{0}^{\circ} \mathrm{C}$ \\
\hline \multicolumn{7}{|l|}{ PP-H/TPE } \\
\hline 0 & - & 35 & 18 & - & 35 & 18 \\
\hline 10 & $1,5 \pm 0,7$ & 47 & 34 & $1,2 \pm 0,7$ & 49 & 43 \\
\hline 20 & $2,4 \pm 0,8$ & 101 & 66 & $1,8 \pm 0,8$ & 107 & 68 \\
\hline 30 & $2,3 \pm 0,8$ & 252 & 129 & $1,6 \pm 0,8$ & $\mathrm{sq}$ & 215 \\
\hline \multicolumn{7}{|l|}{ PP-R/TPE } \\
\hline 0 & - & 51 & 22 & - & 51 & 22 \\
\hline 10 & $1,5 \pm 0,5$ & 75 & 47 & $1,3 \pm 0,3$ & 68 & 38 \\
\hline 20 & $1,8 \pm 0,7$ & 115 & 75 & $1,4 \pm 0,5$ & 164 & 85 \\
\hline 30 & $1,3 \pm 0,6$ & sq & 191 & $1,2 \pm 0,3$ & $\mathrm{sq}$ & $\mathrm{sq}$ \\
\hline
\end{tabular}

sq : sem quebra do corpo de prova

viscosidades entre o elastômero e a matriz ( $\eta *$ TPE $/ \eta$ *PP), a qual para o SEBS (55) foi superior a do SBS (40). Durante a mistura, sendo esta razão alta, a matriz de PP consegue transferir com maior facilidade tensões de cisalhamento para os domínios elastoméricos, promovendo uma melhor dispersão do elastômero. Tal fato pode justificar, de maneira geral, a melhor dispersão ou menor valor do tamanho médio dos domínios elastoméricos do SEBS em ambos polipropilenos. No entanto, uma maior compatibilidade entre os componentes de uma mistura favorece a miscibilidade interfacial matrizdomínios dispersos, estabilizando estes. A maior afinidade química e miscibilidade interfacial entre o PP e o SEBS pode ser relacionada a menor diferença entre os valores dos parâmetros de solubilidade do polipropileno $\left(16,6 \mathrm{~J} / \mathrm{cm}^{3}\right)^{1 / 2} \mathrm{e}$ do copolímero de etileno-butileno $\left(16,2 \mathrm{~J} / \mathrm{cm}^{3}\right)^{1 / 2}$, semelhante ao bloco EB, do que com o polibutadieno $\left(17,6 \mathrm{~J} / \mathrm{cm}^{3}\right)^{1 / 2}$, semelhante ao bloco B. O parâmetro de solubilidade dos blocos de poliestireno é da ordem de $17,6\left(\mathrm{~J} / \mathrm{cm}^{3}\right)^{1 / 2}$ estando este igualmente presente em ambos os $\mathrm{TPEs}^{[26]}$. Assim para as blendas de PP-R com 30\% de TPE (SBS ou SEBS), nas quais o tamanho médio dos domínios são semelhantes, a resistência ao impacto superior da blenda com SEBS se justifica pela maior afinidade química entre o bloco central EB poliolefínico (copolímero(etileno-butileno)) com a região amorfa da matriz, composta principalmente do copolímero(propilenoetileno) e PP atático, ambos de natureza igualmente olefínica. Essa semelhança química favorece interações e interdifusão de segmentos de cadeia na interface matriz-domínios dispersos com diminuição da tensão interfacial, aumentando a adesão entre as fases ${ }^{[18]}$, sendo, portanto, mais efetiva nas blendas de PP com SEBS.

As blendas PP-R/TPE apresentaram melhor resistência ao impacto do que as PP-H/TPE para todas as composições ava- liadas, devido a maior capacidade de interação da matriz com os blocos centrais do TPE. As blendas de PP-R/SEBS apresentaram melhor resposta mecânica observada pela tenacidade dos materiais nos ensaios de impacto em ambas as temperaturas analisadas.

\section{Propriedades mecânicas das blendas PP/TPE}

Verificou-se que o comportamento de tensão-deformação dos polipropilenos PP-H e PP-R e de suas blendas com os TPEs variou em função do tipo de elastômero e da matriz de PP, como pode ser observado no perfil das curvas de tensãodeformação mostradas na Figura 4. A adição progressiva de TPE no polipropileno causa redução na tensão de escoamento e um aumento do alongamento na ruptura.

As blendas de PP-H/TPE apresentaram comportamento de tensão-deformação similar aos encontrados na literatura $^{[14,23,27-31]}$, sendo este uma conseqüência da rigidez do material, dependente, portanto, do teor de cristalinidade do polímero e da sua capacidade de deformação plástica. Os TPEs utilizados continham 30\% de estireno em sua composição, fração esta que se encontra rígida devido à alta temperatura de transição vítrea do bloco poliestirênico, em torno de $100^{\circ} \mathrm{C}$, o que os torna materiais mais rígidos que os elastômeros tipo EPR ou EPDM, normalmente utilizados em blendas com PP [2-6]. A rigidez das blendas PP-H/TPE variou devido à quantidade de TPE adicionada, sendo as composições comparativamente mais rígidas do que aquelas com igual teor de elastômero convencional, conforme descrito na literatura $^{[3,29]}$, devido à fração estirênica do elastômero termoplástico. As blendas PP-R/TPE e PP-H/TPE apresentam comportamento de tensão-deformação diferenciado. Para as blendas de PP-R com 10 a 30\% de TPE não se observou ruptura dos corpos de prova até o limite de extensibilidade 

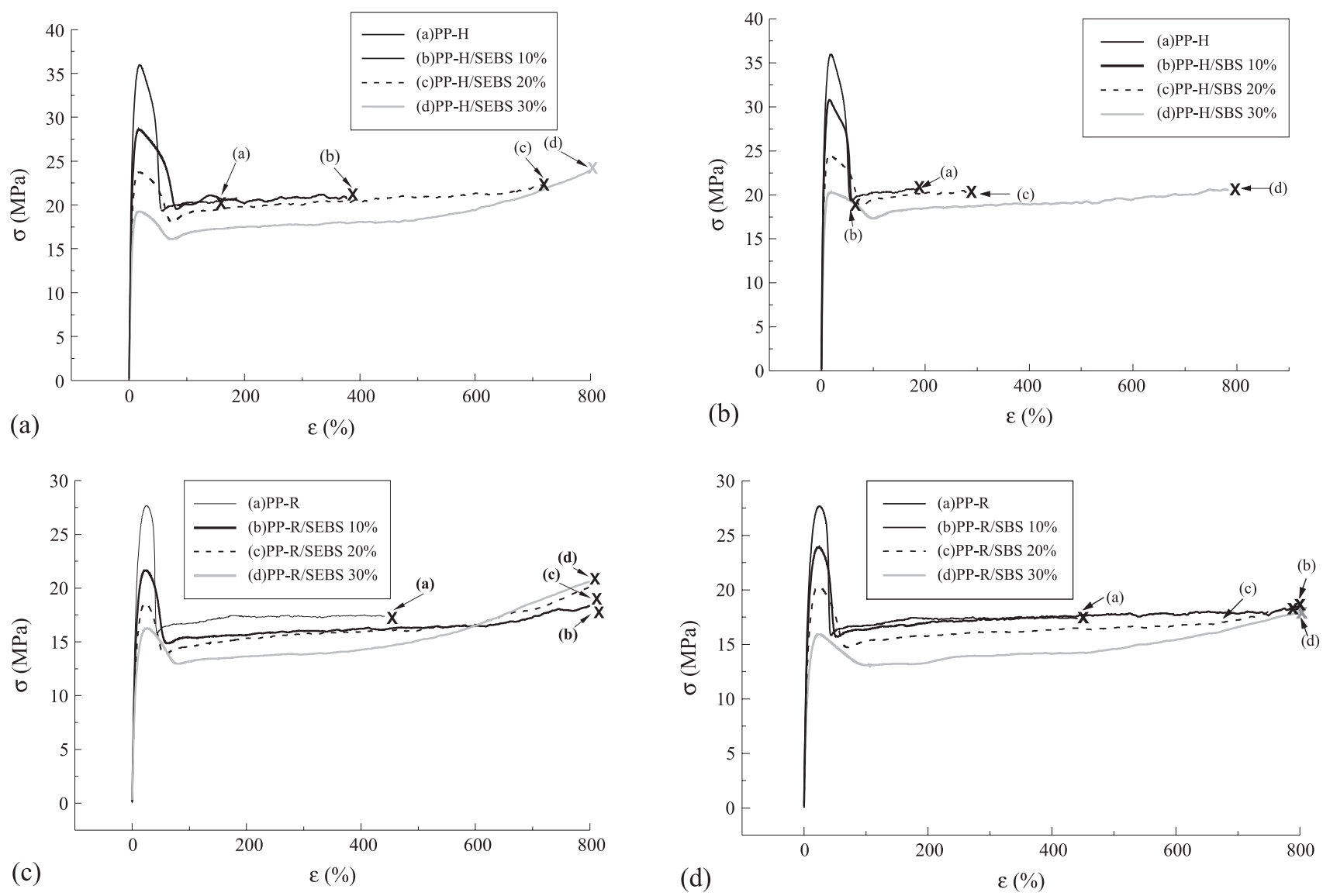

Figura 4. Curvas de tensão-deformação das blendas de PP-H com (a) SEBS e (b) SBS e PP-R com (c) SEBS e (d) SBS.

do equipamento, apresentando, portanto, alto percentual de elongamento. Para o PP-R, um teor de 10\% de TPE é suficiente para produzir um material com excelente tenacidade.

Os valores de cristalinidade dos PPs e das propriedades mecânicas obtidos a partir das curvas de tensão-deformação dos PPs e suas blendas com TPE encontram-se na Tabela 3. O módulo de Young está associado à rigidez do material, e o comportamento na região do escoamento pode ser avaliado pelos valores de tensão e alongamento. A largura do pico de escoamento aumenta gradativamente com o teor de TPE, sendo este aumento mais pronunciado para o SEBS. A blenda PP-H/ SBS10\% apresentou menor elongação na ruptura do que PP puro, piorando a habilidade do material em suportar tensões. Tal fato pode ser atribuído ao maior teor de cristalinidade da matriz de PP-H na blenda com $10 \%$ de SBS (61\%) em relação ao homopolímero de PP (56\%). Conforme discutido em trabalho anterior ${ }^{[21]}$, os TPEs atuaram como agente de nucleação e propiciaram um aumento relativo da cristalinidade da matriz de polipropileno. Outro fator para a menor resistência do material se deve ao maior diâmetro médio dos domínios da fase elastomérica. Na blenda PP-H com 10\% SBS, os domínios estirênicos segregados foram mais efetivos como agente de nucleação, enquanto a interação entre os blocos butadiênicos e a fração amorfa do PP não foi suficiente para permitir uma boa habilidade do material em sofrer deformação plástica, contrapondo o efeito da maior cristalinidade. Assim, a adição de $10 \%$ SBS no PP-H não favorece as propriedades mecânicas da blenda. Tal efeito não foi observado nas demais blendas de PP$\mathrm{H}$ e PP-R com os TPEs, apesar de ter ocorrido um aumento de 3 a $6 \%$ na cristalinidade da matriz de PP nas blendas em relação aos polímeros puros.

A adição de 10\% de TPE causou uma redução dos valores de módulo de Young do PP-H e PP-R em torno de 76 e 61\%, respectivamente. Para teores maiores de elastômero, em ambos os casos, a redução do módulo foi muito similar para as duas matrizes utilizadas. De uma maneira geral, a adição de maiores teores de elastômeros convencionais como EPR ou EPDM na matriz de PP causam uma queda progressiva no módulo ${ }^{[3]}$. No entanto, a adição de teores maiores que $10 \%$ de TPE no PP a diminuição do módulo foi mais atenuada. Este menor efeito sobre o módulo pode ser devido ao fato dos TPEs apresentarem domínios rígidos de poliestireno, os quais atuam como pontos de reticulação da fração elastomérica flexível (bloco central), ou pelo pequeno aumento, de maneira geral, da cristalinidade da matriz de $\mathrm{PP}^{[21]}$. O PP-R tem menor percentual de cristalinidade que o PP-H, portanto apresentou menor módulo de Young e tensão no escoamento, tendo $440 \%$ de alongamento na ruptura contra $180 \%$ do PP-H. As blendas de PP-R/TPE10\% apresentaram grandes deformações, com valores de módulo de Young da mesma ordem de grandeza que os obtidos para as blendas de PP-H/TPE. A Figura 5 mostra a variação do módulo de Young e alongamento na ruptura em função do teor e do tipo de TPE nas blendas com PP-H e PP-R. 
Tabela 3. Propriedades mecânicas das blendas PP/TPE_e cristalinidade do PP

\begin{tabular}{|c|c|c|c|c|c|c|c|c|c|c|c|c|c|c|c|}
\hline \multirow[b]{2}{*}{$\mathrm{PP}$} & \multirow[b]{2}{*}{$\begin{array}{c}\text { TPE } \\
(\%)\end{array}$} & \multicolumn{2}{|c|}{$\begin{array}{l}\text { Módulo Young } \\
\text { (MPa) }\end{array}$} & \multicolumn{2}{|c|}{$\begin{array}{c}\text { Tensão no } \\
\text { escoamento } \\
\text { (MPa) }\end{array}$} & \multicolumn{2}{|c|}{$\begin{array}{c}\text { Alongamento no } \\
\text { escoamento } \\
(\%)\end{array}$} & \multicolumn{2}{|c|}{$\begin{array}{l}\text { Largura do pico } \\
\text { de escoamento }\end{array}$} & \multicolumn{2}{|c|}{$\begin{array}{c}\text { Tensão na } \\
\text { Ruptura } \\
\text { (MPa) }\end{array}$} & \multicolumn{2}{|c|}{$\begin{array}{c}\text { Alongamento na } \\
\text { Ruptura } \\
(\%)\end{array}$} & \multicolumn{2}{|c|}{$\begin{array}{c}\text { Cristalinidade } \\
(\%)\end{array}$} \\
\hline & & SEBS & SBS & SEBS & SBS & SEBS & SBS & SEBS & SBS & SEBS & SBS & SEBS & SBS & SEBS & SBS \\
\hline \multirow[t]{4}{*}{ PPH } & 0 & \multicolumn{2}{|c|}{$1812 \pm 45$} & \multicolumn{2}{|c|}{$36 \pm 0,3$} & \multicolumn{2}{|c|}{$18 \pm 0,1$} & \multicolumn{2}{|c|}{54} & \multicolumn{2}{|c|}{$21 \pm 0,6$} & \multicolumn{2}{|c|}{$180 \pm 66$} & \multicolumn{2}{|c|}{56} \\
\hline & 10 & $439 \pm 12$ & $427 \pm 11$ & $29 \pm 0,1$ & $30 \pm 0,2$ & $17 \pm 0,5$ & $15 \pm 0,5$ & 71 & 55 & $19 \pm 0,5$ & $20 \pm 0,9$ & $388 \pm 99$ & $87 \pm 8$ & 59 & 61 \\
\hline & 20 & $337 \pm 8$ & $379 \pm 15$ & $24 \pm 0,2$ & $24 \pm 0,1$ & $18 \pm 1,0$ & $15 \pm 0,3$ & 70 & 68 & $19 \pm 0,2$ & $21 \pm 0,5$ & $725 \pm 17$ & $288 \pm 17$ & 53 & 58 \\
\hline & 30 & $237 \pm 9$ & $239 \pm 11$ & $19 \pm 0,1$ & $20 \pm 0,2$ & $18 \pm 0,7$ & $17 \pm 0,4$ & 79 & 96 & $21 \pm 0,3^{\mathrm{b}}$ & $26 \pm 0,1^{\mathrm{b}}$ & $843 \pm 9^{\mathrm{b}}$ & $>850^{\mathrm{b}}$ & 53 & 56 \\
\hline \multirow[t]{4}{*}{ PPR } & 0 & \multicolumn{2}{|c|}{$1138 \pm 20$} & \multicolumn{2}{|c|}{$27 \pm 0,3$} & \multicolumn{2}{|c|}{$24 \pm 0,7$} & \multicolumn{2}{|c|}{40} & \multicolumn{2}{|c|}{$20 \pm 1,0$} & \multicolumn{2}{|c|}{$441 \pm 10$} & \multicolumn{2}{|c|}{38} \\
\hline & 10 & $200 \pm 14$ & $276 \pm 17$ & $22 \pm 0,5$ & $24 \pm 0,4$ & $24 \pm 0,4$ & $23 \pm 0,4$ & 58 & 50 & $19 \pm 0,2^{\mathrm{b}}$ & $23 \pm 0,3^{b}$ & $>850^{\mathrm{b}}$ & $>850^{\mathrm{b}}$ & 43 & 44 \\
\hline & 20 & $257 \pm 5$ & $270 \pm 10$ & $19 \pm 0,3$ & $20 \pm 0,2$ & $23 \pm 0,9$ & $23 \pm 0,3$ & 56 & 66 & $22 \pm 0,3^{\mathrm{b}}$ & $23 \pm 0,4^{\mathrm{b}}$ & $>850^{\mathrm{b}}$ & $>850^{\mathrm{b}}$ & 41 & 40 \\
\hline & 30 & $139 \pm 4$ & $141 \pm 7$ & $16 \pm 0,3$ & $16 \pm 0,3$ & $26 \pm 0,7$ & $24 \pm 0,5$ & 77 & 110 & $19 \pm 0,1^{\mathrm{b}}$ & $21 \pm 0,1^{\mathrm{b}}$ & $>850^{\mathrm{b}}$ & $>850^{\mathrm{b}}$ & 43 & 40 \\
\hline
\end{tabular}

a: unidades arbitrárias; b: não sofreu ruptura
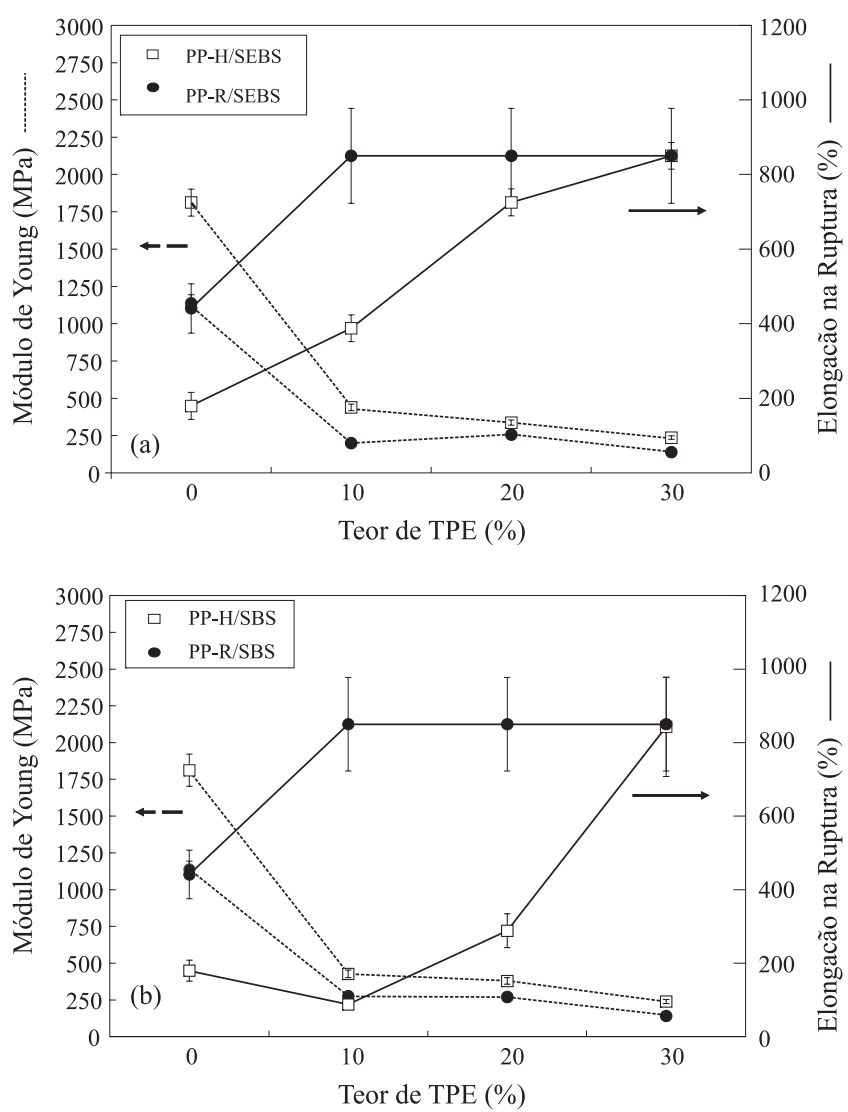

Figura 5. Variação do módulo de Young e da deformação na ruptura em função do tipo de PP e tipo e teor de TPE. (a) PP-H/SEBS e PP-R/SEBS e (b) $\mathrm{PP}-\mathrm{H} / \mathrm{SBS}$ e PP-R/SBS

A adição de SEBS (Figura 5a) e de SBS (Figura 5b) no PP$\mathrm{H}$ e o PP-R tem efeito diferenciado no módulo e no alongamento na ruptura, tanto devido ao tipo de TPE como ao tipo de matriz. Para a matriz PP-R, ambos TPEs mostraram um efeito tenacificante, ao passo que para a matriz PP-H, o SEBS mostrou-se mais eficiente, o que pode ser atribuído a uma maior interação do TPE com a região amorfa do polipropileno. Em suma, a adição de $10 \%$ de TPE na matriz PP-R produz resultados de tração similares aos obtidos com a adição de $30 \%$ de TPE na matriz PP-H, cujas propriedades de tensão no escoamento e módulo de Young são da mesma ordem de grandeza. Particularmente, na blenda PP-R/SEBS10\% houve um sinergismo entre a rigidez do elastômero, e a contribuição deste para um leve aumento da cristalinidade da matriz de PP, e a elasticidade do TPE e a maior fração amorfa do polipropileno para obtenção de um excelente balanço rigidez-impacto.

\section{Conclusões}

As blendas PP/TPE apresentaram domínios elastoméricos diferenciados independente do tipo de TPE e matriz. A resistência ao impacto das blendas PP/TPE variou significativamente com o tipo e o teor de TPE. Para as blendas de PP-R/SEBS30\% não houve fratura dos corpos de prova nas temperaturas de 23 e $0^{\circ} \mathrm{C}$, evidenciando que o TPE nesta quantidade estabiliza as trincas formadas na matriz do PP. A maior resistência ao impacto e a capacidade de deformação das blendas PP-H/SEBS pode ser atribuída além da homogeneidade e do tamanho médio dos domínios elastoméricos a maior similaridade estrutural e química do bloco central do TPE com a matriz de polipropileno. As blendas de PP-R/TPE apresentaram maior capacidade de deformação do que aquelas com PP-H devido a natureza mais elastomérica da matriz. Tanto o polipropileno isotático (PP-H) quanto o polipropileno randômico (PP-R) foram tenacificados pela adição de TPE, podendo estes ser utilizados como modificadores de impacto com bons resultados de propriedades mecânicas, sendo que a blenda PP-R/SEBS10\% apresentou o melhor balanço rigidez-impacto.

\section{Agradecimentos}

Os autores agradecem ao CNPq, pela bolsa de mestrado, e a empresa BRASKEM S.A., pelo apoio laboratorial e analítico. 


\section{Referências Bibliográficas}

1. Kim, G. M.; Michler, G. H.; Gahleitner, M \& Mulhaupt, R. - Polym. Advanced Technologies, 9, p.709 (1998).

2. Silva, A. L. N. \& Coutinho, F. M. B. - Polymer Testing, 15, p. 45 (1996).

3. Wal, A.; Mulder, J. J.; Oderkerk, J. \& Gaymans, R. J. Polymer, 39, p.6781 (1998).

4. Karger-Kocsis, J. \& Csikai, I. - Polym. Eng. Sci,. 27, p.241 (1987).

5. D’Orazio, L.; Mancarella C.; Martuscelli, E.; Cecchin G. \& Corrieri R. - Polymer 40, p. 2745 (1999).

6. Fan, Z; Zhang, Y; Xu, J.; Wang, H \& Feng. L. - Polymer 42, p. 5559 (2001).

7. Kontopoulou, M.; Wang, W.; Gopakumar, T. G. \& Cheung, C.-Polymer 44, p. 7495 (2003).

8. Kim, G. M.; Michler, G. H.; Gahleitner, M. \& Fiebig, J. - J. Appl. Polym. Sci., 60, p.1391 (1996).

9. Yokoyama, Y. \& Ricco, T. - Polymer 39, p. 3675 (1998).

10. Long, Yu \& Shanks, R. A. - J. Appl. Polym. Sci., 61, p.1877 (1996).

11. Heino, M.; Kirjava, J.; Hietaoja, P. \& Seppälä J. - J. Appl. Polym. Sci. 65, p.241 (1997).

12. Bassani, A.; Pessan, L.A. \& Hage, E. - J. Appl. Polym. Sci., 82, p.2185 (2001)

13. Wilhelm, H. M. \& Felisberti, M. I. - J. Appl. Polym. Sci. 85, p.847 (2002).

14. Wilhelm, H. M. \& Felisberti, M. I. - J. Appl. Polym. Sci. 87, p.516 (2003).

15. Chen, J.; Wang, G.; Zeng, X.; Zhao, H.; Cão, D.; Yun, J \& Tan, C. K - J. Appl. Polym. Sci. 94, p.796 (2004).

16. Melo, T. J. A. de; Carvalho, L. H.; Calumby, R. B. et al. Polímeros, 10, p.82 (2000).

17. Hlavatá, D. ; Hromadkova, J.; Fortelny, I. ; Hasová, V. \& Pulda, J. - J. Appl. Polym. Sci. 92, p.2431 (2004).
18. Spontak, R. J.; Williams, M. C. \&. Agard, D. A. - Polymer, 29, p.387 (1988).

19. Abreu, F. O. M. S. - "Blendas de Polipropileno com Elastômeros Termoplásticos tipo SBS e SEBS”, Dissertação de Mestrado, Univ. Federal do Rio Grande do Sul, Brasil (2004).

20. Dywer, S. M.; Boutni, O. N. \& Shu, C. - “ Compounded Polypropylene Products”, in: Polypropylene Handbook, cap 5, Noore Jr., E. P., Hanser Publishers, New York (1996).

21. Abreu, F. O. M. S; Forte, M. M. C. \& Libermann, S. A. J. Appl. Polym. Sci., 95, p.254 (2005).

22. Phillips R. A. \& Wolkowicz, M. D. - "Structure and Morphology" in: Polypropylene Handbook, cap.3 Moore, E. P. Jr. (ed), Hanser Publishers, New York, (1996).

23. Stricker, F.; Thomann Y. \& Mülhaupt R. - J. Appl. Polym. Sci., 68, p.1891 (1998).

24. Tjong, S. C.; Xu S. A. \& Mai, Y.W. - Mat. Sci. and Eng., A347 p.338 (2003).

25. Gupta, A. K \& Purwar, S. N. - J. Appl. Polym. Sci., 29, p. 1595 (1984).

26. Bilmeyer, F. W. - "Textbook of Polymer Science". JohnWiley \& Sons, New York (1984).

27. Saroop, M. \& Mathur, G. N. - J. Appl. Polym. Sci., 71, p.151 (1999).

28. Ferrer, G. G.; Sánchez, M. S.; Sánchez, E. V.; Colomer, F, R \& Ribelles J. L.G. - Polym. Int., 49, p.853 (2000).

29. Yu, T. C. - "Plastomer Selection for Polypropylene Modification", in: Anais do ANTEC'95. p. 2374, Boston, Maio (1995).

30. Gupta, A. K. \& Purwar, S. N. - J. Appl. Polym. Sci., 29, p.3513 (1984).

31. Saroop, M.; \& Mathur, G. N. - J. Appl. Polym. Sci, 65, P.2691 (1997)

Enviado: 01/06/05

Reenviado: 25/10/05

Aprovado: 10/03/06 